\title{
La lithographie par rayons $X$ à très haute résolution - ses limites ultimes
}

A.M. Haghiri-Gosnet

Laboratoire de Microstructures et de Microélectronique, L2M/CNRS, 196 avenue H. Ravera, BP. 107, 92225 Bagneux cedex, France

\begin{abstract}
:
Proximity X-Ray Lithography (XRL) using synchrotron radiation has proven its ability to replicate patterns in the sub-100nm regime, corresponding to the 1 Gigabit integration density for circuits and memories (DRAMs). In that context, the L2M research activities are focused on the ultimate limits in resolution. This paper describes the nanomask technology. It discusses about resolution and replication process latitude in the resist by comparing theorical simulations (L2M code) and very short proximity exposures. $50 \mathrm{~nm}-$ width patterns have been successfully obtained at very small gaps $(\leq 10 \mu \mathrm{m})$. Finally it will be shown that the ultimate resolution should be lower than $20 \mathrm{~nm}$.
\end{abstract}

Résumé: La lithographie aux rayons $\mathrm{X}$ par proximité sur rayonnement synchrotron permet de reproduire des motifs de dimension très inférieure à $100 \mathrm{~nm}$, ce qui correspond à un taux d'intégration des circuits intégrés VLSI ou des mémoires supérieur au Gbit. Nous présentons ici l'activité du groupe "lithographie X" au L2M, dont l'axe principal de recherche est l'étude des limites ultimes en résolution de cette technique. La technologie de fabrication des nanomasques $\mathrm{X}$ est décrite. La résolution et la latitude de procédé d'insolation dans la résine sont discutées par comparaison entre des simulations théoriques (code L2M) et des expériences réalisées en très proche proximité sur rayonnement synchrotron. Des réplications fidèles de traits et de réseaux de dimension latérale $50 \mathrm{~nm}$ ont été réalisées pour des gaps très faibles $(\leq 10 \mu \mathrm{m})$. Enfin on montrera que la résolution ultime par contact (gap=0) doit être inférieure à $20 \mathrm{~nm}$.

\section{INTRODUCTION}

Dans la course à l'intégration des circuits VLSI et des mémoires, on sait que la dimension minimale des motifs doit être inférieure à $180 \mathrm{~nm}$ avec un contôle dimensionnel de $18 \mathrm{~nm}$ et une précision de positionnement de $40 \mathrm{~nm}$ pour atteindre une densité d'intégration du Gigabit[1]. La lithographie $X$ sur rayonnement synchrotron a prouvé qu'elle est compétitive en terme de résolution, de vitesse de production et de latitude de procédé. En effet des mémoires SRAM à $512 \mathrm{kBits}$ dans une technologie CMOS à $250 \mathrm{~nm}$ ont été fabriquées par IBM[2] et sont étudiées par NTT[3]. Récemment des HEMTs de longueur de grille $150 \mathrm{~nm}$ ont été réalisés au L2M[4] et des MOSFETs de grille 80nm au MIT[5]. 
Indépendamment des applications en microélectronique, la résolution ultime de la lithographie $X$ reste un sujet de débat et constitue un des axes principaux de recherche au L2M. Ainsi nous avons implanté une station de lithographie autour de l'anneau Super ACO à Orsay équipée d'un photorépéteur commercial (Karl Suss XRS 200) et mis au point une technologie de nanomasques $X$ adaptés[6].

\section{LES NANOMASQUES $X$}

La raison majeure, pour laquelle les industriels sont réticents à changer d'infrastructure technologique (de l'UV aux RX), est la complexité de fabrication des masques X. Les masques $X$ sont " $1 X$ ". Ceci signifie que les motifs doivent être dessinés sur le masque à la même dimension que celle qui sera reproduite dans la résine. Les matériaux de fabrication utilisés doivent être choisis en fonction de critères sévères décrits ci-dessous. L'optimisation de l'écriture des motifs en lithographie électronique et leur transfert dans l'absorbant sont aussi des points clés.

Un masque est constitué d'une fine membrane ( 1 à $2 \mu \mathrm{m})$ en matériau léger suffisamment transparent aux RX qui supporte des motifs métalliques absorbants minces $(\approx 0.5 \mu \mathrm{m})$. Les matériaux pour membranes les plus intéressants en terme de résistance à la fracture, de rigidité de stabilité sous rayonnement et de transparences $\mathrm{X}$ et optique sont le $\mathrm{SiC}$ [7] et le diamant[8]. L'absorbant est un métal à grand $\mathrm{Z}$ comme $\mathrm{Au}, \mathrm{W}$ et Ta.

Une première technologie en $\mathrm{W} / \mathrm{SiC}$ a permis de réaliser des nanomasques adaptés au photorépéteur[9]. Le tungstène est déposé par pulvérisation rf magnétron avec des paramètres de dépôt permettant d'obtenir une structure dense et stable dans le temps avec une contrainte interne minimale[10]. Une couche de résine PMMA est ensuite insolée à $50 \mathrm{kV}$ sous faisceau d'électrons (système d'écriture JEOL 5D2U) pour générer les motifs. Ceux-ci sont transférés dans le W par gravure ionique réactive (SF6/CHF3) à basse température[11]. Des nanomasques porteurs de réseaux de traits de taille minimale 50nm (Figure 1) ont été réalisés. Pour diminuer la taille des traits afin d'étudier la très haute résolution, une seconde technologie est en cours d'étude. Un faisceau d'électrons d'énergie élevée 200kV (STEM Philips CM20) est utilisé pour l'écriture dans des conditions minimales de taille de sonde $(\approx l n m)$. Dans ce cas, l'insolation se fait sur petites membranes de SiC pour minimiser l'effet des électrons rétrodiffusés[12]. La technologie consiste à faire croître de l'Au par électrodéposition dans les motifs de résine. Les premiers masques obtenus sont porteurs de traits aussi petits que $30 \mathrm{~nm}$, comme le montre la figure 2 .

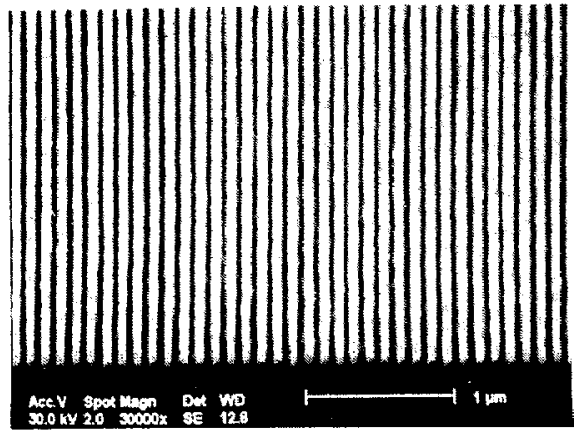

Fig.1.Réseaux de traits de $50 \mathrm{~nm}$ au pas de $120 \mathrm{~nm}$ en tungstène (épaisseur $300 \mathrm{~nm}$ ) obtenus sur des membranes de $3 \mathrm{~cm}^{2}$.

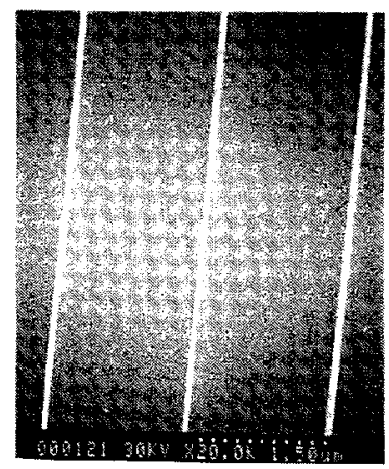

Fig.2. Réseaux de traits de 30nm en $\mathrm{Au}$ (épaisseur $200 \mathrm{~nm}$ ) réalisés avec une écriture à $200 \mathrm{kV}$ sur membranes $\mathrm{SiC}$. 


\section{LES LIMITES ULTIMES EN RESOLUTION}

Lorsqu'un système utilise une optique de focalisation, la taille minimale du trait qu'il est possible de répliquer dans la résine, et, par là même, la résolution, est fixée par l'ouverture numérique en sortie du système optique. C'est le cas des systèmes lithographiques travaillant dans le visible et l'U.V. Pour la lithographie aux rayons $\mathrm{X}$, dite à proximité, il n'y a pas de système de focalisation entre le masque porteur des motifs et l'échantillon recouvert de résine. La résolution est alors purement limitée par deux facteurs: la diffraction au travers du masque $\mathrm{X}$ et le libre parcours des photoélectrons émis dans la résine.

\subsection{Les limites en diffraction:}

La formation de l'image derrière le masque $\mathrm{X}$ s'étudie en utilisant la théorie de diffraction en champ proche. Considérons la diffraction sur un trait de largeur $W$ et sur un réseau de traits de même largeur et de périodicité p. La figure 3 illustre les images obtenues à une distance $G$ (gap) du masque, éclairé par un rayonnement $X$ de longueur d'onde $\bar{\lambda}$. Notons ici que $\bar{\lambda}$ est la moyenne de la distribution spectrale $\Delta \lambda$ de la source si celleci n'est pas monochromatique. Ces images aériennes, représentées en trait plein sur la figure 3, sont en fait les distributions spatiales de l'intensité normalisée I/Io du champ diffracté.

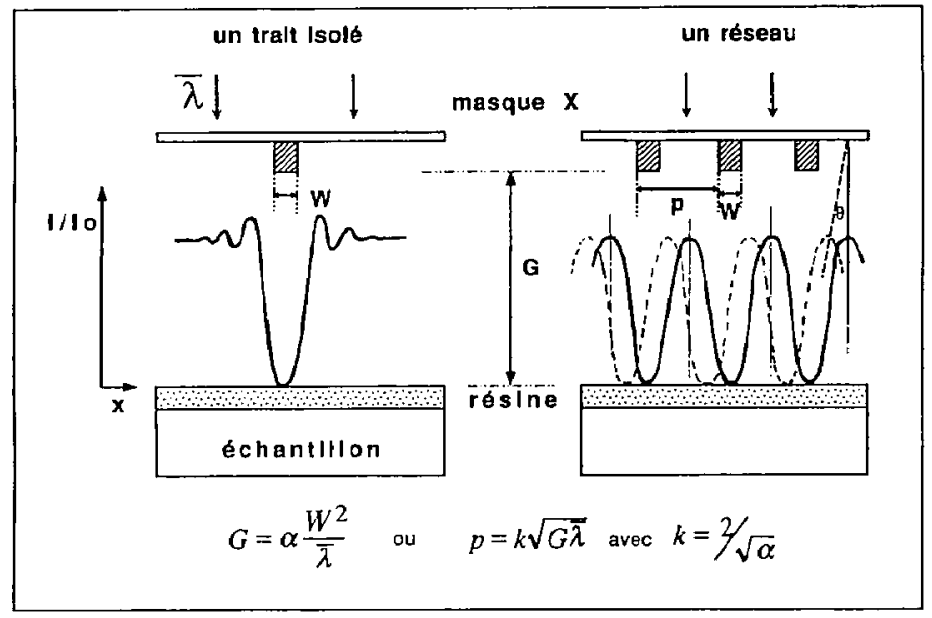

Figure 3. La formation d'images aériennes de diffraction en très proche proximité

En première approrhe, la latitude de procédé en gap peut s'écrire avec les formules simples:

$\begin{array}{ll}\text { (1) } G=\alpha \frac{W^{2}}{\bar{\lambda}} \text { avec le paramètre } \alpha \leq \alpha \max & \text { pour tout type de motif } \\ \text { (2) } p=k \sqrt{G \bar{\lambda}} \text { avec le paramètre } k \geq k \text { min } \quad \text { pour un réseau }\end{array}$

L'équation (1) montre que la taille du motif sera minimale si $\bar{\lambda}$ et $G$ sont petits. Le plus grand gap utilisable, sans perte de la résolution, est donné par la valeur maximale du paramètre $\alpha$. En se basant sur des calculs simples, on a pensé pendant longtemps que $\alpha$, qui est l'inverse du carré du nombre de Fresnel, ne pouvait pas dépasser la valeur $\alpha_{\max }=0,4$. En 1991, W. Chu et al[13], réussirent à répliquer dans du PMMA un trait de $50 \mathrm{~nm}$ jusqu'à $\mathrm{G}=2,72 \mu \mathrm{m}$ avec $\lambda \mathrm{CuL}=1.3 \mathrm{~nm}$, ce qui correspond à une valeur de $\alpha_{\max }$ élevée $\left(\alpha_{\max }=1,44\right)$. Cette expérience démontre que la latitude de procédé de la lithographie $\mathrm{X}$ est beaucoup plus grande que ce que prédit la théorie de Fresnel. 
L'équation (2) exprime la période minimale qui peut être résolue pour un réseau de traits. Ceci correspond à une modulation nulle dans le plan image, lorsque l'intensité diffractée d'ordre 1 (courbe en pointillé sur la figure ) est décalée d'une demi-période par rapport à l'intensité diffractée d'ordre zéro. La valeur du paramètre $\mathrm{k}$ dépendante du gap est reliée à $\alpha$ par $k=2 \sqrt{ } \alpha$, et doit être minimisée.

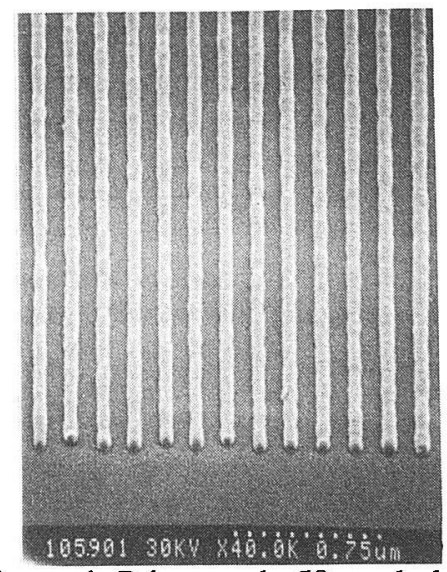

Récemment le groupe de lithographie du L2M[14] a répliqué des réseaux de $50 \mathrm{~nm}$ au pas de 160nm dans du PMMA sur rayonnement synchrotron $\bar{\lambda}=0.65 \mathrm{~nm}$ et sur photo répéteur à très faible gap contrôlé $\mathrm{G}=5 \mu \mathrm{m}$. Ces motifs sont visibles sur l' image ci-contre (photo MEB). Ces expériences montrent que $\mathrm{k}_{\min }=\sqrt{ } 3=1,73$, soit $\alpha_{\max }=1,33$. Elles confirment le résultat de Chu et al. pour un rayonnement nonmonochromatique à large bande.

Figure 4. Réseaux de $50 \mathrm{~nm}$ de large de période $160 \mathrm{~nm}$ obtenus dans PMMA épais de $200 \mathrm{~nm}$ à $\mathrm{G}=5 \mu \mathrm{m}$.

Le calcul exact des images aériennes a été fait pendant longtemps avec des méthodes scalaires, basées sur les intégrales de diffraction de Rayleigh-Sommerfeld (RS) ou de Fresnel. Dans les deux cas, les conditions aux limites appliquées étaient celles de Kirchoff (KBC). KBC suppose que les composantes du champ électromagnétique sont totalement découplées des équations de Maxwell. Ceci veut dire que le champ (en amplitude et en phase) est constant dans les régions transparentes entre les motifs absorbants, et qu'il est aussi constant mais atténué et déphasé sous les motifs. Donc le profil en intensité du champ à $\mathrm{G}=0$ ne présente pas d'oscillations à chaque transition absorbant/non-absorbant et ressemble à une fonction créneau. En fait on peut utilisé KBC si $\bar{\lambda}$ est très inférieure à la taille du motif diffractant $W$ et si le motif a une épaisseur suffisante pour absorber complètement les rayons $\mathrm{X}$. Ces deux conditions ne sont pas remplies en nanolithographie $X$ puisque l'absorbant est un diélectrique épais de 10 à 100 fois $\bar{\lambda}$ et que W est inférieur à $100 \mathrm{~nm}$. La méthode BPM (Beam Propagation Method) permet de calculer la valeur exacte du champ pas à pas dans le motif absorbant tout au long de la propagation du faisceau. Ensuite le champ calculé à $\mathrm{G}=0$ (juste sous les motifs) est transféré dans le plan image (surface de la résine) par une méthode classique de diffraction. Dans la pratique on utilise des méthodes de calcul vectoriel, pour lesquelles la seule approximation est la discrétisation de l'espace objet et des équations de Maxwell. Cette discrétisation étant faite à une échelle suffisamment petite par rapport à $\bar{\lambda}$, ces calculs vectoriels sont extrêmement précis [15-17].

La différence majeure entre l'image aérienne exacte calculée par BPM et celles calculées par les méthodes scalaires réside en la présence d'oscillations parasites sur les images scalaires. Le calcul scalaire suppose que toutes les fréquences spatiales sont contenues dans le champ transmis par le masque (illumination cohérente spatialement). Les fréquences supérieures à $0.05 \mathrm{~nm}^{-1}$ sont à l'origine des oscillations parasites[16]. La figure 5 montrent les images obtenues par les différentes méthodes pour un trait isolé de $50 \mathrm{~nm}$ de large et épais de 300nm (absorbant tungstène) à deux gaps différents $\mathrm{G}=0,384 \mu \mathrm{m}(\alpha=0,1)$ et $\mathrm{G}=3,84 \mu \mathrm{m}(\alpha=1)$. La suppression des hautes fréquences parasites est d'autant plus visible que le gap $G$ est petit (faible valeur de $\alpha$ ) comme le montre la figure 5a). Dans ce cas, le calcul BPM est indispensable. Pour des valeurs plus élevées de $\alpha$ ( $\alpha=1$ fig.5b) il y a toujours désaccord entre les méthodes scalaires (Fresnel 
et RS) et BPM. Ceci a été démontré par M.L. Schattenburg et al[15] pour des valeurs de $\alpha$ allant jusqu'à 1,5. On comprend ici l'utilité de la méthode BPM et la grande latitude en gap expérimentale $\left(\alpha_{\max }=1,44\right)$ démontrée précédemment.
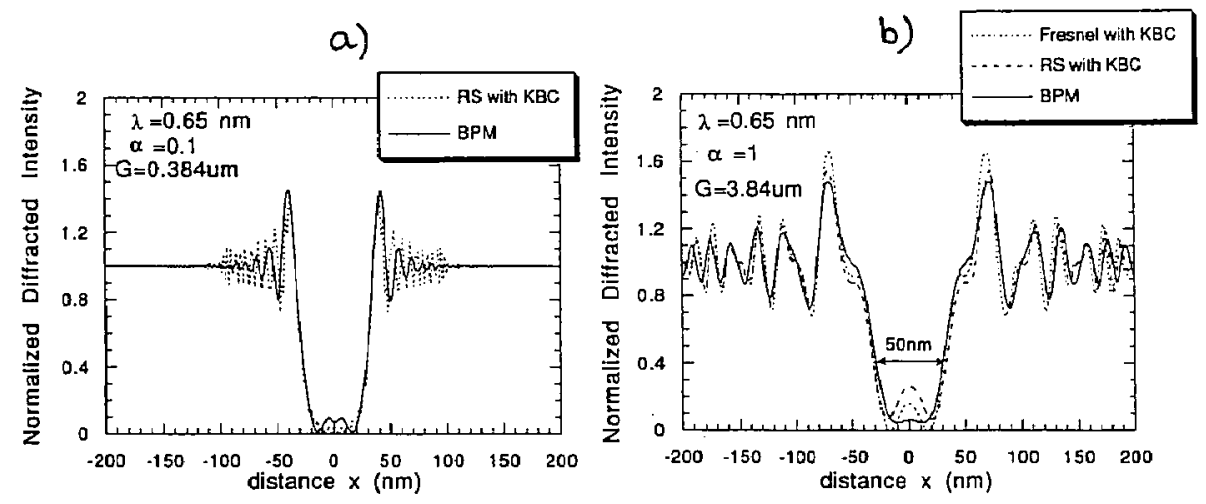

Figure 5. Images aériennes calculées par BPM et par les méthodes scalaires pour un trait isolé de $50 \mathrm{~nm}$ de large et épais de $300 \mathrm{~nm}$ (matériau absorbant tungstène) pour une source monochromatique $\lambda=0.65 \mathrm{~nm}$
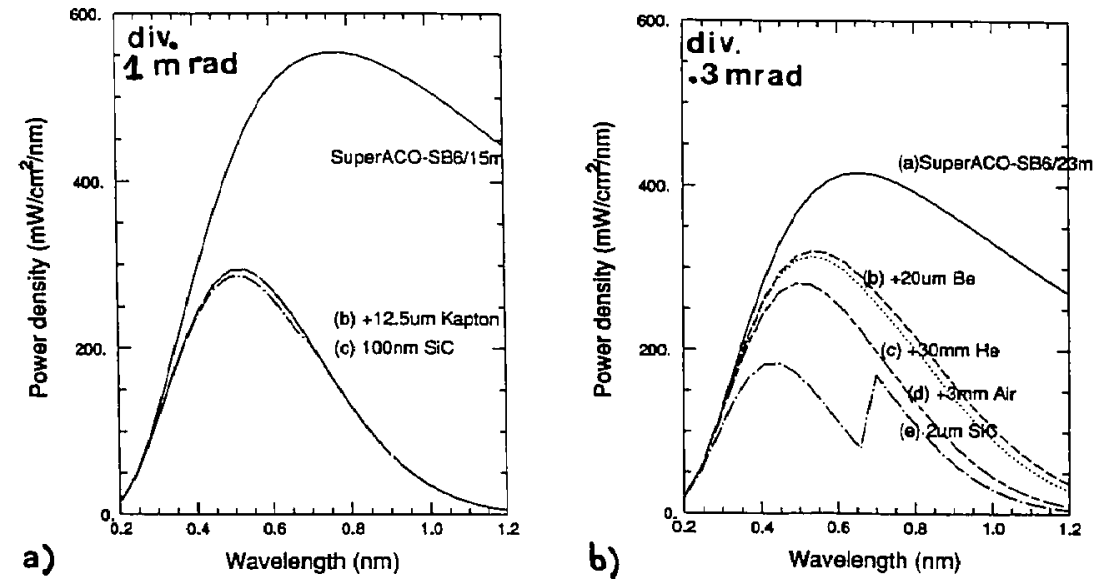

Figure 6. Distributions spectrales utilisées sur la station de lithographie du L2M sur l'anneau Super-ACO a) à 15 mètres dans la chambre pour expériences en contact et b) à 23 mètres sur le photo répéteur Karl Suss

Dans le cas d'une source synchrotron, la distribution spectrale doit être prise en compte. La station de lithographie du L2M est installée sur l'anneau de stockage Super-ACO à Orsay. Elle possède une chambre à vide pour effectuer des réplications en contact $(\mathrm{G}=0)$ et un photo répéteur placé en ligne à 23 mètres de la source pour les réplications à faibles gaps $(5 \mu \mathrm{m}<\mathrm{G}<40 \mu \mathrm{m})$. La figure 6 donne les deux distributions en longueurs d'onde correspondantes: a) celle qui correspond à une divergence verticale maximale de $1 \mathrm{mrad}$ derrière un seul filtre de $12 \mu \mathrm{m}$ en kapton et un masque en $\mathrm{SiC}$ mince $(100 \mathrm{~nm})$, b) celle tenant compte de tous les éléments absorbants (Be, $\mathrm{He}$, air) au bout de la ligne derrière le photo répéteur aveć des masques plus épais (esiC=2 $=2 \mu \mathrm{m}$ ).

Pour effectuer un calcul complet et précis des images aériennes, il faut tenir compte de la distribution spectrale de la source et de sa divergence spatiale, de la présence de tous les 
filtres existants dans la ligne de lumière mais aussi du caractère diélectrique imparfait de l'absorbant et du fait que le motif peut avoir des flancs inclinés.

L'ensemble des calculs présentés sur la figure 7 montre l'influence de chacun de ces effets. La figure 7a) montre qu'une distribution à large bande est préférable à une lumière monochromatique: les oscillations parasites sont quasi-inexistantes. D'autre part il a été montré qu'un certain degré d'incohérence spatiale de la source améliore aussi l'image[18]: la courbe thérique correspondant à une divergence spatiale de $6 \mathrm{mrad}$ a été calculée pour comparaison et ne montre qu'une légère amélioration de l'image. Notons ici que des méthodes sophistiquées, comme la vibration ou l'inclinaison de l'ensemble masqueéchantillon, ont été proposées pour diminuer la cohérence spatiale des sources synchrotron, dont la divergence naturelle est de l'ordre du mrad. La figure 7b) montre l'effet de décalage de phase du champ au travers du motif absorbant. La qualité de l'image et son contraste dépendent beaucoup de l'épaisseur de l'absorbant. Elle doit être de l'ordre de $300 \mathrm{~nm}$ pour notre exemple d'un trait de $50 \mathrm{~nm}$ en tungstène. Sur la figure $7 \mathrm{c}$ ) l'effet de forme du motif, c'est à dire l'écart à la verticalité des flancs, est présenté: un écart de $4^{\circ}$ qui correspond à une différence de $20 \mathrm{~nm}$ entre le haut et le bas du motif de $50 \mathrm{~nm}$ ne semble pas ici altérer l'image. Ainsi la taille minimale qu'on réussira à reproduire sera liée à la largeur du motif à mi-hauteur et non à sa forme.
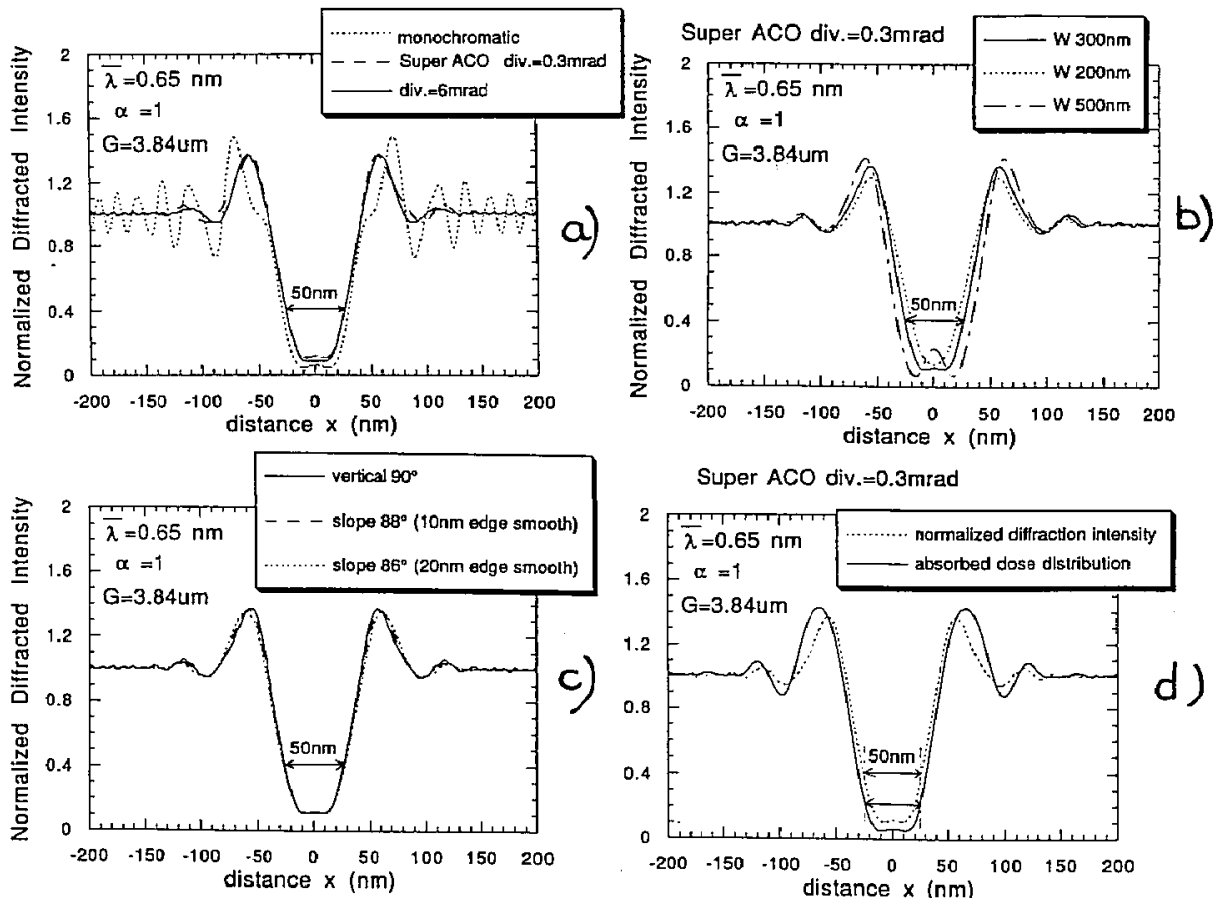

Super ACO div. $=0.3 \mathrm{mrad}$

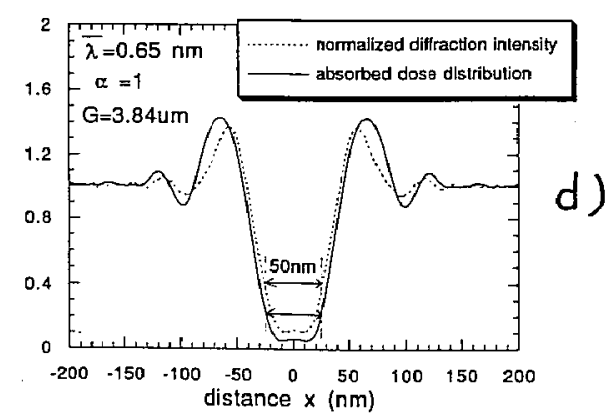

Figure 7. Influences a) d'un spectre large et d'une grande divergence spatiale de la source, b) de l'épaisseur de l'absorbant, c) de la forme du motif. d) comparaison entre l'image aérienne de diffraction et la distribution en dose absorbée dans la résine. 
Enfin, dans le cas d'une source non-monochromatique, il faut tenir compte de la variation d'absorption de la résine en fonction de $\lambda$. Y.Chen [19] a proposé de calculer une distribution en dose absorbée dans la résine peu épaisse. Ce calcul est aujourd'hui le plus réaliste. Par exemple la figure $7 \mathrm{~d}$ ) montre le cas de la réplication d'un trait de $50 \mathrm{~nm}$. Cette distribution est plus large que l'image aérienne puisque le poids relatif des grandes longueurs d'onde est plus important.

\subsection{Effet des photoélectrons:}

Lorsque le masque et le substrat recouvert de résine sont en contact $(\mathrm{G}=0)$, le phénomène limitant la résolution est l'effet des photoélectrons. Un photon $X$ d'énergie voisine de $2 \mathrm{keV}(\bar{\lambda}=0.65 \mathrm{~nm})$ est absorbé au niveau d'un atome de résine provoquant l'émission d'un photoélectron primaire d'énergie relativement élevée (1 keV), ainsi que des électrons secondaires. Pour les résines à faible $\mathrm{Z}$, il s'agit essentiellement des électrons Auger du C $(0.29 \mathrm{keV})$ et de $\mathrm{O}(0.53 \mathrm{keV})$. La densité volumique en dose efficace des électrons Auger est plus grande d'un facteur 10 par rapport à celle des photoélectrons primaires, bien que le libre parcours des électrons Auger soit très petit. La zone insolée par les photoélectrons a été calculée par Occola [20] comme étant inférieure à $5 \mathrm{~nm}$ et attribuée uniquement aux électrons Auger du carbone et de l'oxygène. Pour obtenir une estimation précise de la résolution ultime, il faut aussi tenir compte des photoélectrons réémis par le substrat: un calcul très récent a montré que la zone insolée est du même ordre de grandeur, soit approximativement 3 d $4 \mathrm{~nm}$ [21] que celle calculée dans la résine. Dans ce contexte, une résolution ultime voisine de $10 \mathrm{~nm}$ peut être envisagée.

Il est donc pertinent de calculer l'intensité du champ juste sous les motifs à $G=0$ par la méthode BPM, voire même la distribution en dose absorbée. La figure 8 montre par exemple la possibilité de transférer un trait isolé de $20 \mathrm{~nm}$ (vertical ou très incliné) sur rayonnement synchrotron. Ceci n'a pas encore été démontré expérimentalement. Les meilleures expériences en terme de résolution sont les suivantes: avec des sources monochromatiques ( $\lambda \mathrm{CuL}$ et $\lambda \mathrm{AlK}$ ) des traits de $30 \mathrm{~nm}$ ont été répliqués [22].

La grande profondeur de champ de la lithographie X par proximité, couplée à la haute résolution, a été de plus démontrée au L2M à plusieurs reprises. La figgure 9 montre qu'il est possible de reproduire des motifs de résolution inférieure à $50 \mathrm{~nm}$ avec un facteur de forme très élevé (rapport de la hauteur sur la largeur du motif supérieur à 10).
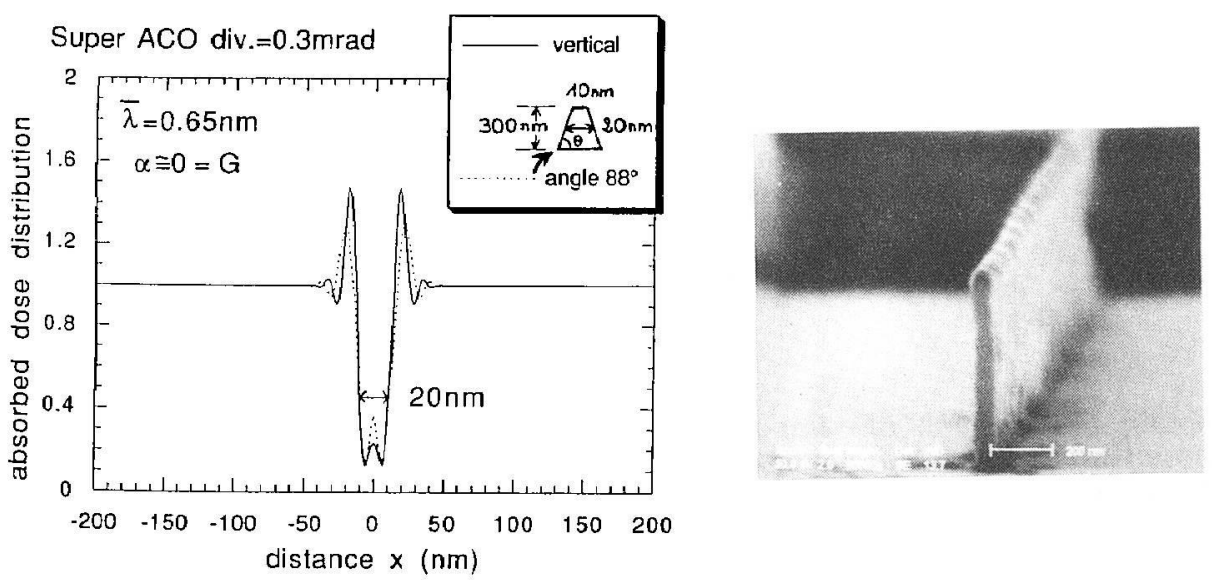

Fig. 8 Distribution en dose à $\mathrm{G}=0$ pour

Fig.9 Motif en PMMA répliqué en contact un trait isolé de $20 \mathrm{~nm}$ de large en tungstène. à Super ACO-hauteur $650 \mathrm{~nm} /$ largeur $60 \mathrm{~nm}$ 


\section{Remerciements:}

Je tiens à remercier vivement mes collègues F.Rousseaux et $Y$. Chen pour nos discussions fructueuses en commun et spécialement $Y$. Chen pour l'utilisation de son code de simulation.

\section{Références bibliographiques:}

[1] M. Peckerar, J. Maldonado, Proceedings of the IEEE, vol. $81 \mathrm{n}^{\circ} 9$ (1993) 1249

[2] R. Viswanathan, A. Brigth et al., J.Vac.Sci.Technol.B11(1994) 2910

[3] K. Deguchi et al., J.Vac.Sci.Technol.B10 (1993) p 3145

[4] A.M. Haghiri-Gosnet, H. Lafontaine et al, Microelectronic Eng. 23 (1994) 243

[5] $\mathrm{H}$. Hu, Isabel Y. Yang et al, the 38th Intern. Symp. on Electron, Ion and Photon Beams,June 1994-New Orleans, to be published in J.Vac.Sci.Technol. Dec.94

[6] F. Rousseaux, A.M. Haghiri-Gosnet, Y. Chen et al, Jpn. J. Appl. Phys. vol 31 (1992) 4520

[7] A.M. Haghiri-Gosnet, F. Rousseaux et al, J.Vac.Sci.Technol.B8 (1990) 1565

[8] M.F. Ravet et al, Mat. Res. Soc. Symp. Proc. vol 306 (1993) 103

[9] R. Rousseaux et al, Conf."Frontiers in Science and Technology with Synchrotron radiation", Avril 94, à paraitre dans les Editions de Physique.

[10] A.M. Haghiri-Gosnet, F.R. Ladan et al, J.Vac.Sci.Technol.A7 (1989) 2663

[11] A.M. Haghiri-Gosnet et al, JJAP series3 vol 3 (1990) 89

[12] M. A. McCord, R. Viswanathan et al, J.Vac.Sci.Technol.B10 (1992) 2764

[13] W. Chu, Henry I. Smith et M.L. Schattenburg, Appl. Phys. Lett. 59 (1991) 1641

[14] Y. Chen et al, the 38th Intern. Symp. on Electron, Ion and Photon Beams,June

1994-New Orleans, to be published in J.Vac.Sci.Technol. Dec.94

[15] M.L. Schattenburg et al, J.Vac.Sci.Technol.B9 (1991) 3232

[16] Scott Hector, M.L. Schattenburg et al, J.Vac.Sci.Technol.B10 (1992) 3164

[17] J. Z. Y. Guo et al, J.Vac.Sci.Technol.B10 (1992) 3150

[18] J. Z. Y. Guo et al, J.Vac.Sci.Technol.B8 (1990) 1551

[19] Y. Chen, R. K. Kupka, F. Rousseaux, Microelectronic Eng. 23 (1994) 239

[20] L.E. Occola, F Cerrina, J.Vac.Sci.Technol.B11 (1993) 2839

[21] L.E. Occola, F Cerrina, the 38th Intern. Symp. on Electron, Ion and Photon Beams,June 1994-New Orleans, to be published in J.Vac.Sci.Technol. Dec.94

[22] K. Early, M.L. Schattenburg et al, Microelectronic Eng. 17 (1992) 149 